\section{Rheological Characterization of Streptomyces roseosporus for the Production of Daptomycin}

\author{
I. Chakravarty, S. Singh, and S. Kundu*
}

School of Biochemical Engineering,

Indian Institute of Technology (Banaras Hindu University),

Varanasi - 221005, India doi: 10.15255/CABEQ.2016.966

Original scientific paper

Received: August 12, 2016

Accepted: July 25, 2017

Daptomycin is a potential broad-spectrum anti-MRSA antibiotic produced by Streptomyces roseosporus. It has been a boon for patients with complicated skin and skin structure infections. There is a constant urge to enhance the yield of Daptomycin to reduce the cost of its industrial production. Daptomycin fermentation is a highly aerobic process, and the fermentation broth exhibits typical rheological properties, which peculiarly govern the production phenomenon. Being a secondary metabolite, Daptomycin is a non-growth associated product. Sufficient cell mass promises better production. The cell growth is particularly affected by stress conditions in the broth. In this study, the effects of stress conditions on broth characteristics were evaluated. Different rheological models were plotted and the Power law model was found to be best-fitted. The consistency index $(K)$ and the flow behavior index $(n)$ data were calculated. This study should contribute to predict and control the rheology of large-scale Daptomycin fermentation.

Key words:

Streptomyces roseosporus, daptomycin, rheological models, fermentation, broth viscosity

\section{Introduction}

Daptomycin has gained importance as a secondary metabolite with a unique mode of action against a wide range of aerobic and anaerobic gram-positive bacteria, including methicillin-resistant Staphylococcus aureus (MRSA) and vancomycin-resistant Enterococci. Streptomyces roseosporus is an important soil actinomycetes for the production of this novel antibiotic, which is the first approved member of the A21978C family of the cyclic anionic 13-amino acid lipopeptide ${ }^{1}$. The morphological differentiation of $S$. roseosporus in culture broth is closely associated with Daptomycin production. Under nutrient limiting conditions and environmental stress conditions, $S$. roseosporus produced spores during the production of Daptomycin in a submerged culture. The morphology of $S$. roseosporus is characterized by prominent features like spore chains, germinating spores, vegetative mycelium, and aerial hyphae ${ }^{2}$. The structural characteristics observed before the addition of precursor showed that mycelium of cells was elongated and thick at 24 hours without Daptomycin being synthesized. It then changed to a slender and apical form after production of Daptomycin at 192 hours. The

"Corresponding author: Email: subirbhu@gmail.com, skundu.bce@itbhu.ac.in; Tel.: +917080185543 addition of decanoic acid increases the accumulation of Daptomycin, which suppresses the cell growth and concludes in structural disruption. The observed morphological changes were coherent with the reduced cell mass after stationary phase. Daptomycin is a non-growth associated product of idiophase. Sufficient biomass ensures better production. The cell growth is particularly affected by stress conditions in the broth. The study of rheological properties influenced by morphological changes in the fermentation broth is of industrial importance, and is indispensable to enhance the yield of the desired product as it depends on the biomass cultured $^{3}$. The filamentous nature of actinomycetes leads to viscous fermentation broths with shear thinning nature ${ }^{4}$. The structural changes of cell wall may be closely linked with the resistance to shear ${ }^{5,6}$. To enhance Daptomycin production, morphological characteristics and stimulation factors in the fermentation process should be simultaneously considered to control the flow behavior of culture broths. There remains the need to eloquently establish the relationship between broth properties and productivity of Daptomycin. In this study, rheological models were compared to delineate the broth characteristics of $S$. roseosporus NBRC 12910, which governs the production of the crucial secondary metabolite, Daptomycin. 


\section{Materials and methods}

\section{Strain}

S. roseosporus NBRC 12910 was used for this study.

\section{Medium and culture conditions}

Streptomyces roseosporus was cultivated and maintained at $30{ }^{\circ} \mathrm{C}$ and $200 \mathrm{rpm}$ in culture broth medium containing $\left(\mathrm{g} \mathrm{L}^{-1}\right)$ : malt extract 3 , glucose 10 , yeast 3 , and peptone 5 at $\mathrm{pH} 6.5$. Fermentation was carried out in a medium containing $\left(\mathrm{g} \mathrm{L}^{-1}\right)$ : dextrin 30, glucose 10, soybean flour 20, Fe $\left(\mathrm{NH}_{4}\right)_{2} \mathrm{SO} 40.6, \mathrm{KH}_{2} \mathrm{PO}_{4} 0.2$, and $\mathrm{pH} 7$, and incubated at $30^{\circ} \mathrm{C}$ for 6 days. Cofactors were added to the sterile culture medium after aseptic filtration. The cultures were shaken in $250 \mathrm{~mL}$ Erlenmeyer flasks at $200 \mathrm{rpm}$ for 6 days. N-decanoic acid was fed at $48 \mathrm{~h}$ after inoculation $\left(0.2 \mathrm{~g} \mathrm{~L}^{-1}\right)$. All experiments were done in triplicate. The cultures were agitated with different numbers of 3-mm borosilicate glass beads (Sigma Aldrich) to expose the microorganism to stress conditions.

\section{Evaluation of the rheology of cells}

The consequence of the stress conditions subjected by the glass beads on the microbial cells was assessed by various rheological model plots. The rheological properties were measured using the Brookfield viscometer. The Non-Newtonian behavior of the microbial cell mass was predicted using the following fluid models.

\section{Newtonian Model: $\tau=n \gamma$}

As per the definition of a Newtonian fluid, the shear stress, and the shear rate are proportional to each other.

\section{Bingham: $\tau-\tau_{\mathrm{yB}}=\mu_{\mathrm{p}} \gamma$}

Newtonian model and the Bingham plastic model can be described by straight lines in terms of shear rate and shear stress. The viscosity and yield stress can be found out by calculating the slope and the intercept.

\section{Casson: $\tau^{0.5}=\left(\tau_{\mathrm{y}, \mathrm{C}}\right)^{0.5}+\mu_{\mathrm{P}, \mathrm{C}} \gamma 0.5$}

In Casson model, straight line results when the square root of shear rate is plotted against the square root of shear stress. The Casson yield stress is calculated as the square of the intercept, and the Casson plastic viscosity as the square of the slope.

\section{Power law: $\tau=K \gamma^{n}$}

Shear stress-shear rate plots of many fluids become linear when plotted on double logarithmic coordinates and the power law model describes the data of shear-thinning and shear thickening fluids.

In the equations, $\tau$ denotes shear stress, $\gamma$ shear rate, $\tau_{\mathrm{y}, \mathrm{B}}$ Bingham yield stress, $\mu_{\mathrm{p}}$ plastic viscosity, $\tau_{\mathrm{y}, \mathrm{C}}$ Casson yield stress, $\mu_{\mathrm{PC}}$ Casson viscosity, $K$ consistency index, $n$ flow behavior (power-law) index ${ }^{7}$.

\section{Results and discussion}

\section{Morphological alterations}

The behavior of the microorganism in submerged culture is dictated by several crucial factors, such as the type of microorganism, media, and culture conditions ${ }^{8-10}$. The growth of the microorganism and hence the metabolite of interest are greatly affected $^{11}$. The typical morphological characteristics of $S$. roseosporus with a wide variety of differentiation patterns were observed in baffled flasks with glass beads as shown in Figure 1. The dispersed swollen hyphae and arthrospores could be witnessed in the culture broth ${ }^{12}$. The increasing viscosity of the broth led to non-Newtonian nature, which is typical of actinomycetes. When the pattern of shear stress varying with shear rate was plotted, shear stress seemed to increase with increasing shear rate. The control culture containing no glass beads revealed lower shear stress, which increased slowly from day 1 to day 6 when hyphae started to differentiate. The shear stress in cultures that include maximum glass beads rose sharply, as depicted in Figure 2.

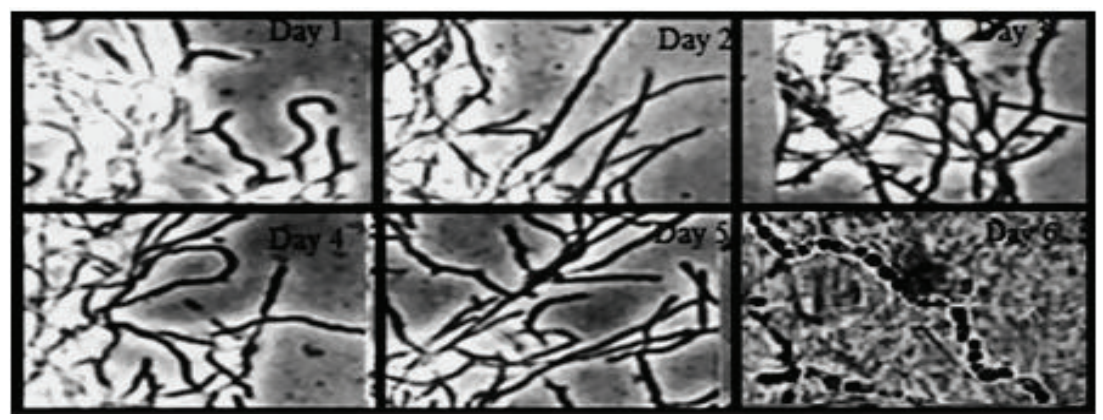

Fig. 1 - Typical morphology of S. roseosporus at different time intervals 


\section{Rheological patterns of $S$. roseosporus}

The pattern depicted in Figure 2 clearly shows the non-Newtonian nature of the microbial culture broth. The change in the pattern can be correlated with the differentiation of the microbial cells with the passing of time and the increasing stress conditions. The plots of the shear stress versus the shear rate of culture broths revealed that the culture broth had a non-Newtonian nature and shear stress increased with the glass beads. The stress level grad-

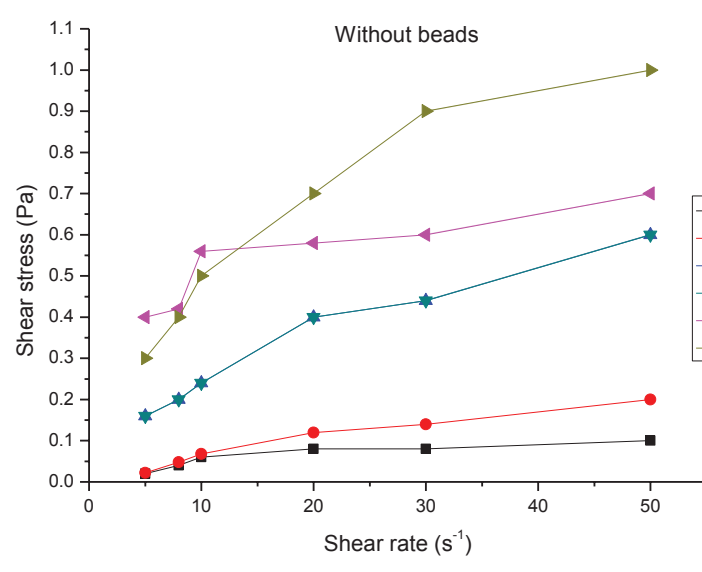

(a)

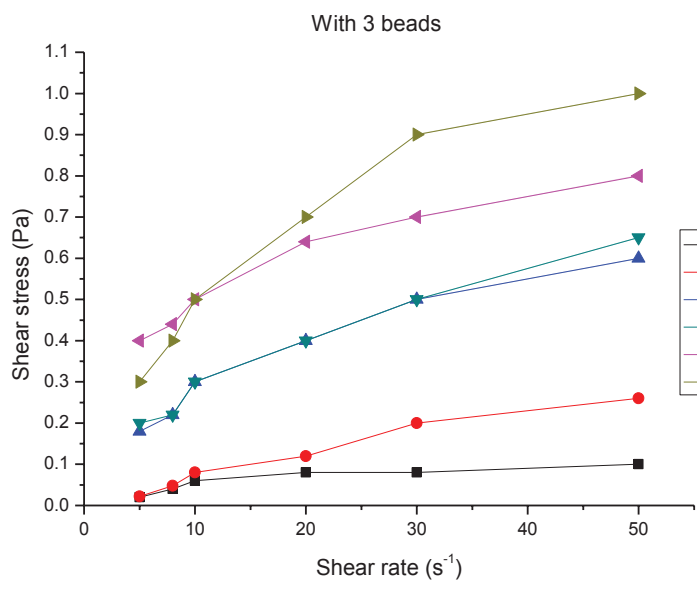

(b)

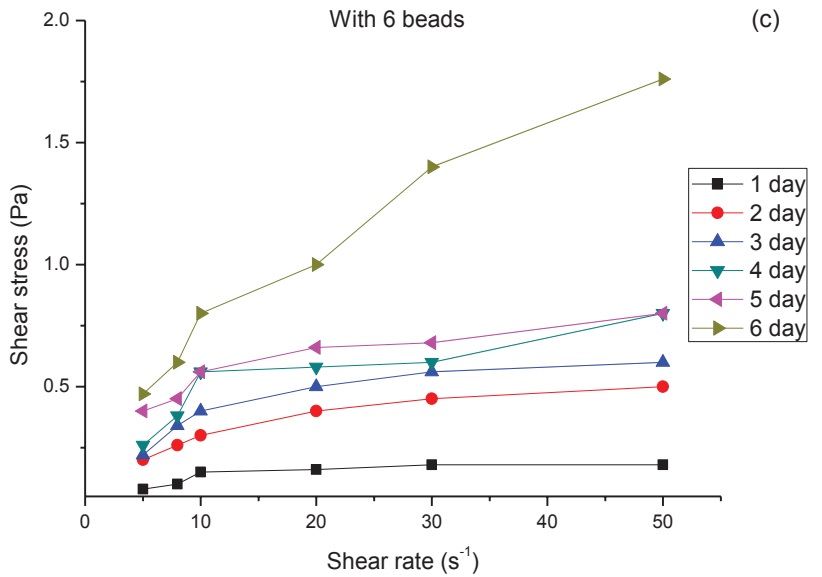

Fig. 2 - Rheological pattern of $S$. roseosporus when grown without beads (a), with 3 beads (b), and with 6 beads (c) in submerged culture broth ually increased as growth and differentiation of cells took place until the differentiation of hyphal structures into arthrospores. The shear rate increased abruptly depending upon the shear rate, which was dominated by the structural changes in the microorganism. The exponential phase marked by cellular differentiation led to a sharp increase in the stress level. Further, different rheological models were plotted to fit the rheological pattern obtained in order to evaluate the non-Newtonian behavior of the fluid (Figures 3 to 6).

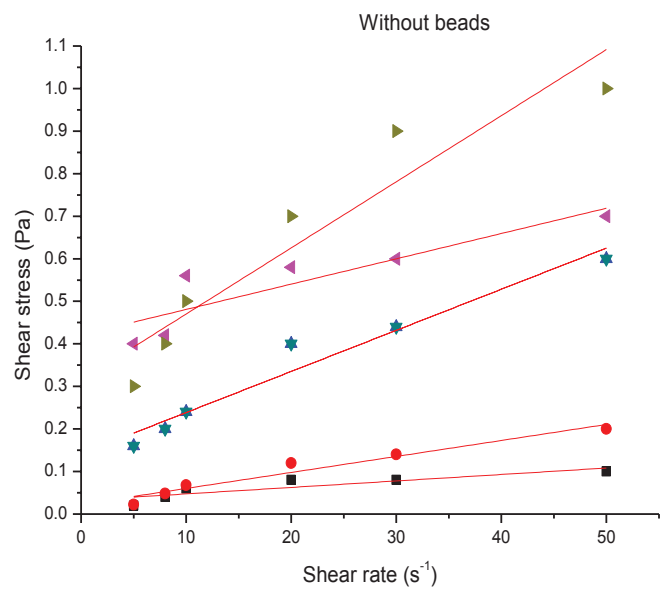

(a)
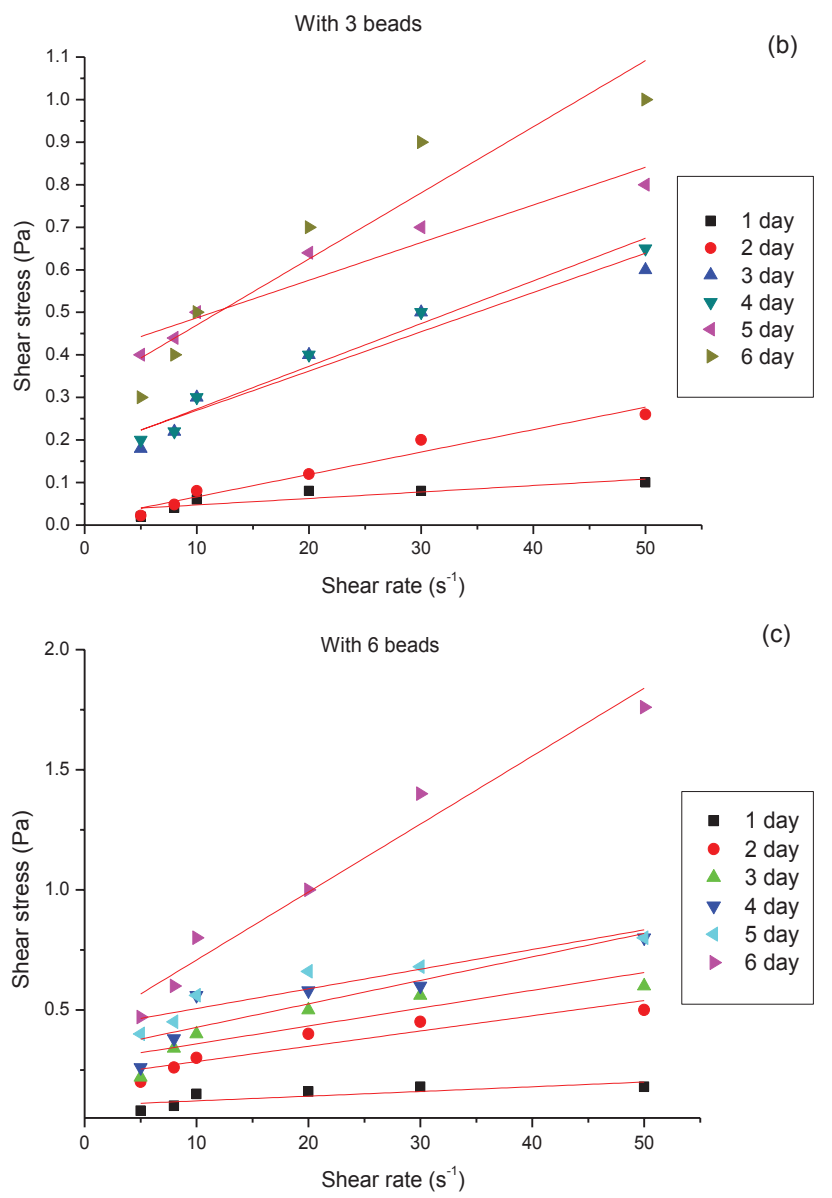

Fig. 3 - Rheological data as predicted by Bingham model when cells grown without beads (a), with 3 beads (b), and with 6 beads (c) 


\section{Bingham plastic model}

In Figure 3, the recorded data was predicted by model fitting experiment wherein the Bingham plastic model based on linear regression fitted the experimental data well until day 5 , but no evidence of curve fitting was witnessed for day 6.

In Figure 3, the recorded data was predicted by model fitting experiment wherein the Bingham plastic model based on linear regression fitted the experimental data well until day 5, but no evidence of curve fitting was witnessed for day 6 .

The parameters obtained from Bingham model and the $R^{2}$ values obtained have been sorted in
Table 1, which reveal that at increased shear rate, the data showed irregular curve fitting.

\section{Casson plastic model}

Casson model showed better fitting than Bingham model, as represented in Figure 4.

Figure 4 represents the shear stress-shear rate plots of the culture broths as determined by the Casson model, showing that the Casson model had an edge over Bingham plastic model for prediction of the cultivation of $S$. roseosporus. The parameters obtained from the model and the $R^{2}$ values are depicted in Table 2, which show that at increased

Table 1 -Bingham model parameters for cell grown without beads, with 3 beads, and 6 beads

\begin{tabular}{|c|c|c|c|c|c|c|c|c|c|}
\hline \multirow{2}{*}{ Day } & \multicolumn{3}{|c|}{$\begin{array}{l}\tau_{\mathrm{y}, \mathrm{B}} \\
\mathrm{Pa}\end{array}$} & \multicolumn{3}{|c|}{$\begin{array}{c}\mu_{\mathrm{P}} \\
\mathrm{P}\end{array}$} & \multicolumn{3}{|c|}{$R^{2}$} \\
\hline & $\begin{array}{c}\text { Without } \\
\text { beads }\end{array}$ & $\begin{array}{l}\text { With } \\
3 \text { beads }\end{array}$ & $\begin{array}{l}\text { With } \\
6 \text { beads }\end{array}$ & $\begin{array}{c}\text { Without } \\
\text { beads }\end{array}$ & $\begin{array}{c}\text { With } \\
3 \text { beads }\end{array}$ & $\begin{array}{l}\text { With } \\
6 \text { beads }\end{array}$ & $\begin{array}{l}\text { Without } \\
\text { beads }\end{array}$ & $\begin{array}{c}\text { With } \\
3 \text { beads }\end{array}$ & $\begin{array}{l}\text { With } \\
6 \text { beads }\end{array}$ \\
\hline 1 & 0.0015 & 0.0322 & 0.1015 & 0.0322 & 0.0015 & 0.002 & 0.782 & 0.782 & 0.634 \\
\hline 2 & 0.0226 & 0.0137 & 0.2214 & 0.001 & 0.0053 & 0.0064 & 0.9502 & 0.9607 & 0.8703 \\
\hline 3 & 0.1419 & 0.1772 & 0.2843 & 0.0097 & 0.0092 & 0.0074 & 0.9568 & 0.9341 & 0.7834 \\
\hline 4 & 0.135 & 0.1726 & 0.3295 & 0.0097 & 0.01 & 0.0098 & 0.9833 & 0.9715 & 0.7946 \\
\hline 5 & 0.436 & 0.3987 & 0.4237 & 0.0054 & 0.0088 & 0.0082 & 0.856 & 0.9199 & 0.8668 \\
\hline 6 & 0.3148 & 0.3148 & 0.4248 & 0.0155 & 0.0155 & 0.0283 & 0.9006 & 0.9006 & 0.9649 \\
\hline
\end{tabular}

Table 2 - Casson model parameters for cell grown without beads, with 3 beads, and with 6 beads

\begin{tabular}{|c|c|c|c|c|c|c|c|c|c|}
\hline \multirow{2}{*}{ Day } & \multicolumn{3}{|c|}{$\begin{array}{c}\tau \\
\mathrm{Pa}\end{array}$} & \multicolumn{3}{|c|}{$\stackrel{\mu_{\mathbf{P}, \mathbf{C}}}{\mathrm{P}}$} & \multicolumn{3}{|c|}{$R^{2}$} \\
\hline & $\begin{array}{l}\text { Without } \\
\text { beads }\end{array}$ & $\begin{array}{l}\text { With } \\
3 \text { beads }\end{array}$ & $\begin{array}{l}\text { With } \\
6 \text { beads }\end{array}$ & $\begin{array}{l}\text { Without } \\
\text { beads }\end{array}$ & $\begin{array}{l}\text { With } \\
3 \text { beads }\end{array}$ & $\begin{array}{l}\text { With } \\
6 \text { beads }\end{array}$ & $\begin{array}{l}\text { Without } \\
\text { beads }\end{array}$ & $\begin{array}{c}\text { With } \\
3 \text { beads }\end{array}$ & $\begin{array}{l}\text { With } \\
6 \text { beads }\end{array}$ \\
\hline 1 & 0.012544 & 0.012544 & 0.066049 & 0.000961 & 0.000961 & 0.000729 & 0.804 & 0.804 & 0.720 \\
\hline 2 & 0.002809 & 0.000289 & 0.131769 & 0.003364 & 0.005329 & 0.002704 & 0.947 & 0.906 & 0.925 \\
\hline 3 & 0.057121 & 0.079524 & 0.1681 & 0.006084 & 0.005625 & 0.003249 & 0.975 & 0.981 & 0.840 \\
\hline 4 & 0.142884 & 0.271441 & 0.298116 & 0.009025 & 0.003025 & 0.002601 & 0.944 & 0.981 & 0.840 \\
\hline 5 & 0.322624 & 0.142884 & 0.178084 & 0.001521 & 0.009025 & 0.017161 & 0.833 & 0.964 & 0.911 \\
\hline 6 & 0.142884 & 0.012544 & 0.066049 & 0.009025 & 0.000961 & 0.000729 & 0.944 & 0.944 & 0.976 \\
\hline
\end{tabular}

Table 3 -Power law model parameters for cells grown without beads, with 3 beads, and with 6 beads

\begin{tabular}{|c|c|c|c|c|c|c|c|c|c|}
\hline \multirow{2}{*}{ Day } & \multicolumn{3}{|c|}{$n$} & \multicolumn{3}{|c|}{$\begin{array}{c}k \\
\mathrm{~Pa} \mathrm{~s}^{\mathrm{n}}\end{array}$} & \multicolumn{3}{|c|}{$R^{2}$} \\
\hline & $\begin{array}{l}\text { Without } \\
\text { beads }\end{array}$ & $\begin{array}{c}\text { With } \\
3 \text { beads }\end{array}$ & $\begin{array}{l}\text { With } \\
6 \text { beads }\end{array}$ & $\begin{array}{l}\text { Without } \\
\text { beads }\end{array}$ & $\begin{array}{c}\text { With } \\
3 \text { beads }\end{array}$ & $\begin{array}{c}\text { With } \\
6 \text { beads }\end{array}$ & $\begin{array}{c}\text { Without } \\
\text { beads }\end{array}$ & $\begin{array}{c}\text { With } \\
3 \text { beads }\end{array}$ & $\begin{array}{l}\text { With } \\
6 \text { beads }\end{array}$ \\
\hline 1 & 0.6252 & 0.6252 & 0.348 & 0.010 & 0.0102 & 0.06 & 0.8352 & 0.8532 & 0.8042 \\
\hline 2 & 0.9034 & 1.0332 & 0.399 & 0.06 & 0.542 & 0.11 & 0.9431 & 0.9515 & 0.9692 \\
\hline 3 & 0.5869 & 0.535 & 0.4083 & 0.0619 & 0.0783 & 0.13 & 0.9881 & 0.977 & 0.8944 \\
\hline 4 & 0.5869 & 0.5295 & 0.4181 & 0.0619 & 0.082 & 0.16 & 0.9881 & 0.9808 & 0.8343 \\
\hline 5 & 0.2328 & 0.3148 & 0.2956 & 0.2816 & 0.239 & 0.25 & 0.8638 & 0.988 & 0.9469 \\
\hline 6 & 0.5379 & 0.5379 & 0.5716 & 0.134 & 0.134 & 0.19 & 0.976 & 0.976 & 0.9829 \\
\hline
\end{tabular}


stress level, the experimental data fitted the Casson model better than the Bingham model.

\section{Power law model}

The rheological data fitted the power law model in the best possible manner, and the fitting was better than the other two mathematical models to predict the non-Newtonian behaviour of the broth.

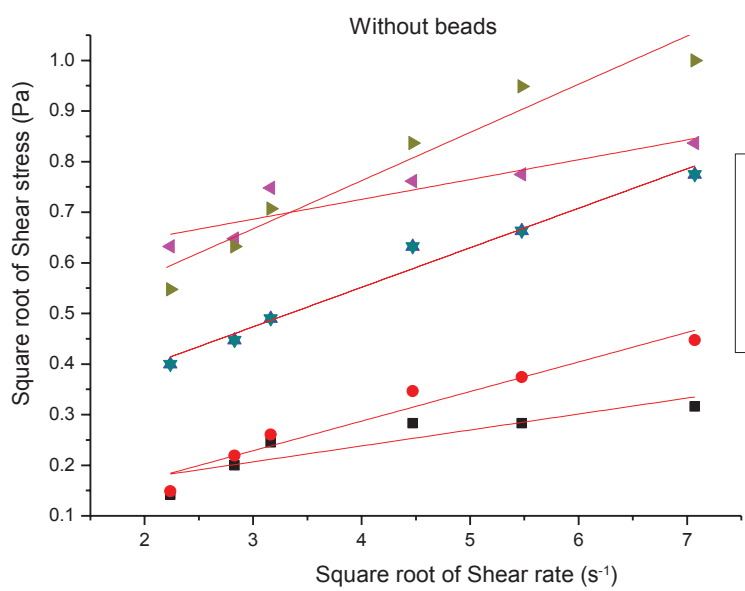

(a)
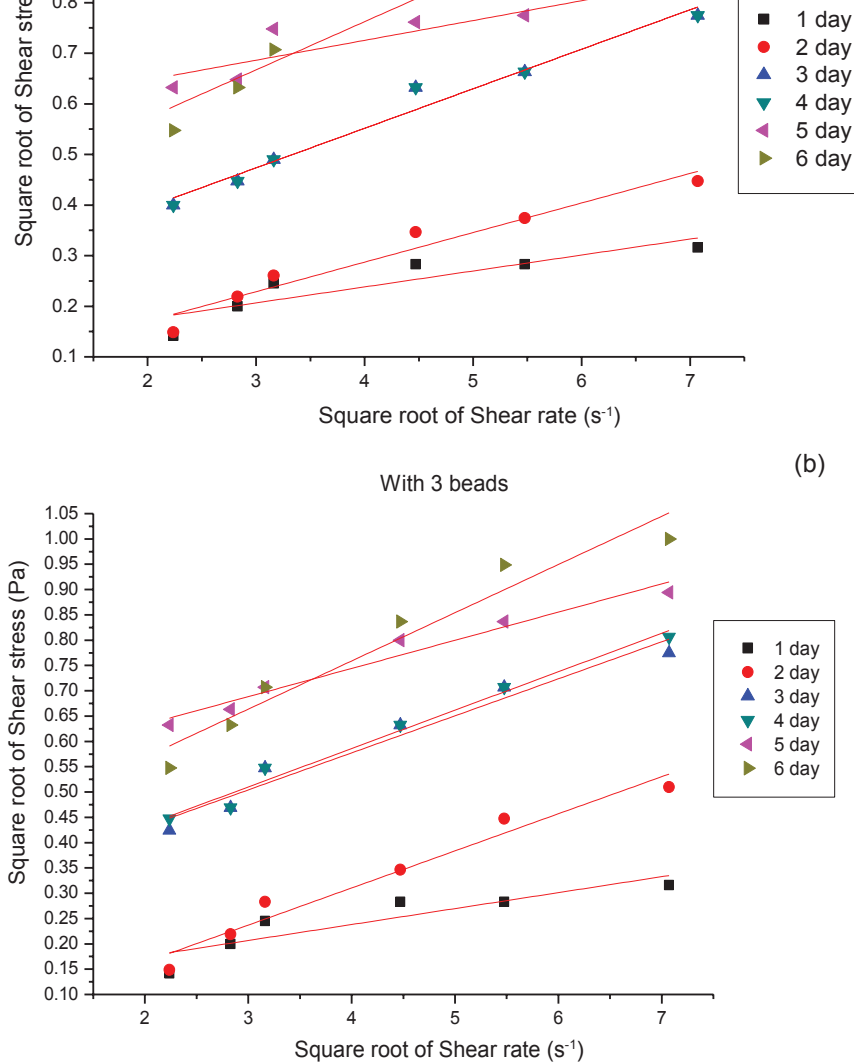

(b)

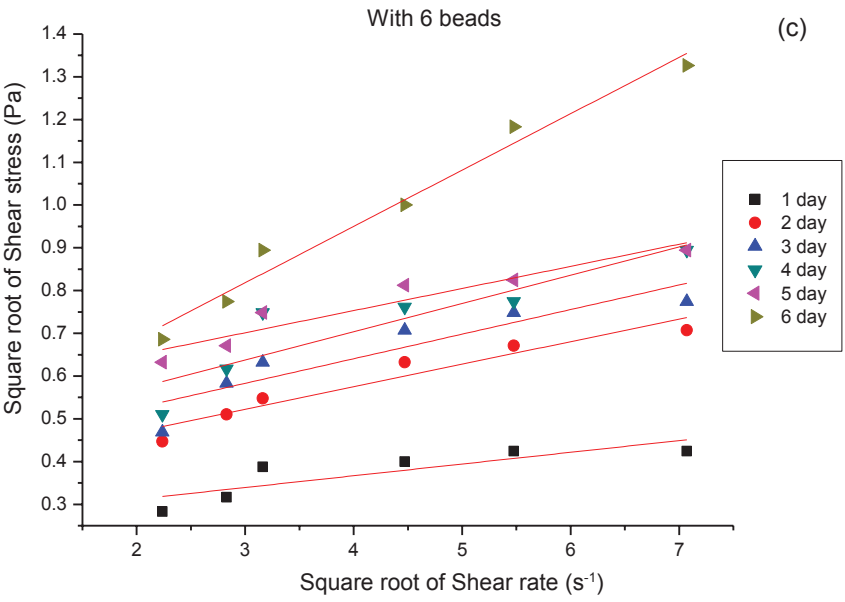

Fig. 4 - Rheological data as predicted by Casson model when cells grown without beads (a), with 3 beads (b), and 6 beads (c)
The power law model has been quite significant in chemical and biological transport systems. Figure 5 proves that the curves adapted with the experimentally recorded data fairly well. The parameters obtained from the model and the $R^{2}$ values are represented in Table 3, which confirm that the experimental data fits this mathematical model convincingly.

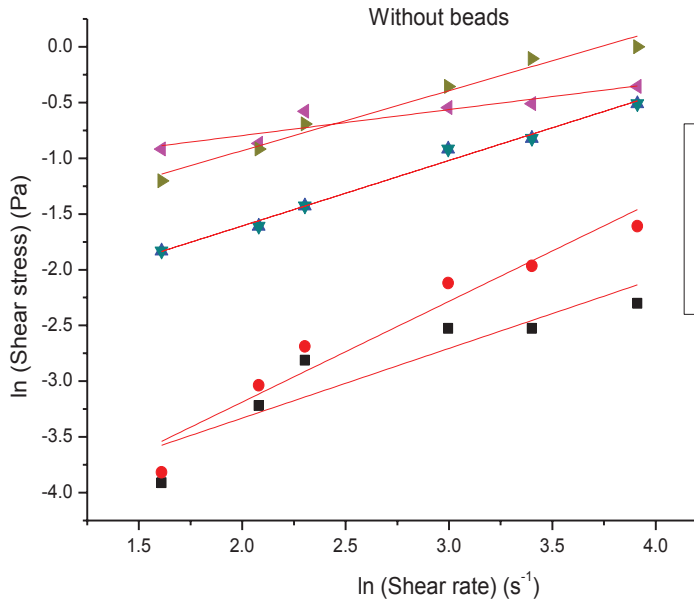

(a)

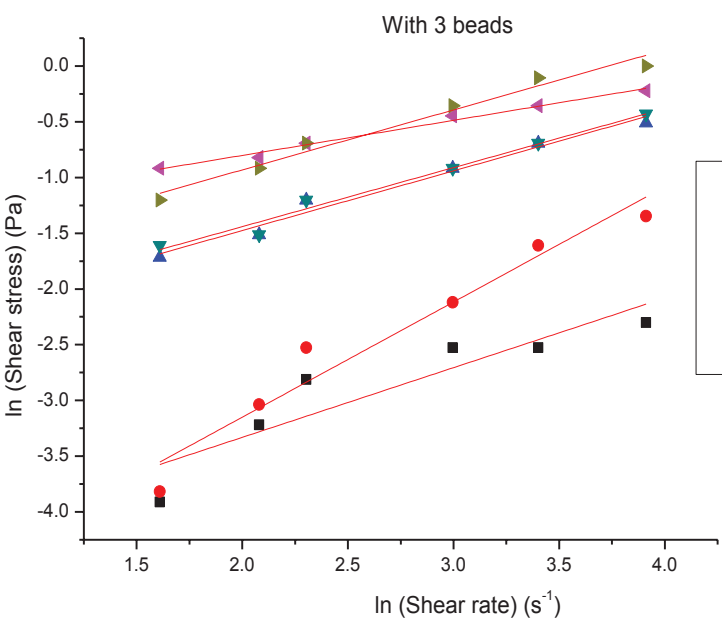

(b)

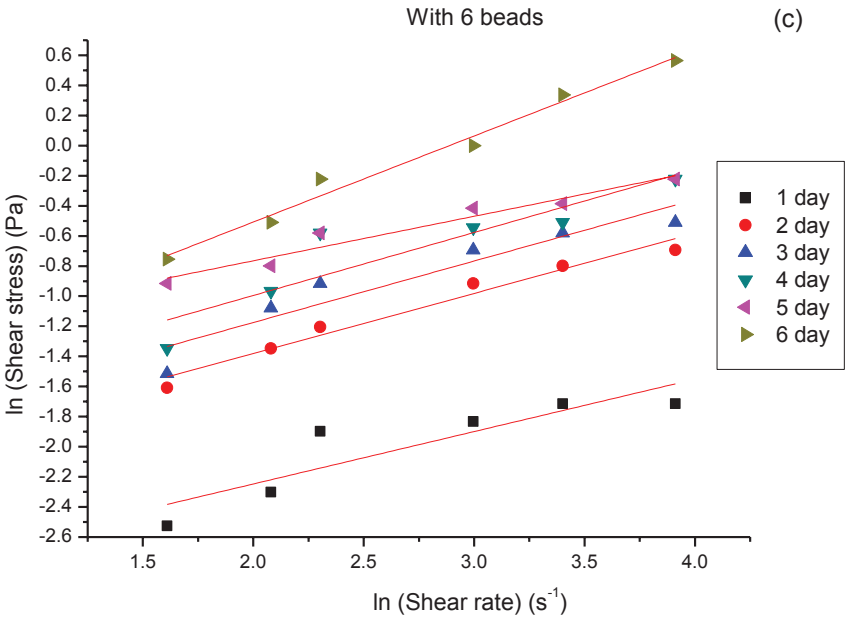

Fig. 5 - Rheological data as predicted by the Power law model for cells grown without beads (a), with 3 beads (b), and 6 beads (c) 


\section{Parameters of best fitted model}

The power law model depicted the rheological patterns of the culture broths of $S$. roseosporus fairly better than the Bingham plastic model. It was also observed that the consistency index $(K)$ and the flow behavior index $(n)$ of the power-law fluid model prompted the morphological variations with increasing stress level, demonstrating that the culture broth becomes more shear-thinning with increasing stress (Figure 6). Since the power law model fitted the data well, the consistency index $(K)$ and the flow behavior index $(n)$ of the power-law fluid model were taken into consideration to show the shear-thinning nature of the culture broth with increasing stress (Figure 7).

The consistency index $(K)$ for $S$. roseosporus without glass beads increased and then decreased. With increasing stress, the culture broth portrayed a significant increment in $K$ and $n$ with time. On the contrary, the power-law index $(n)$ decremented as stress increased, revealing the fact that the broth

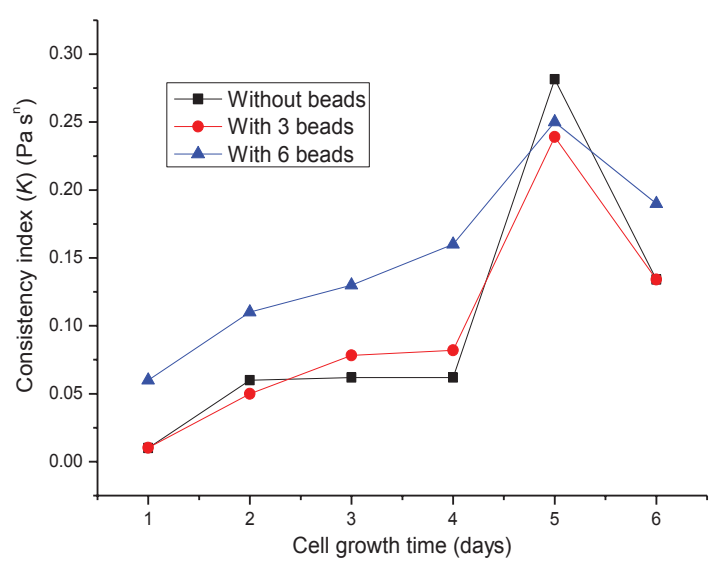

Fig. 6 - Variations in consistency index $(K)$ of the power-law fluid model as cell growth occurs with or without glass beads

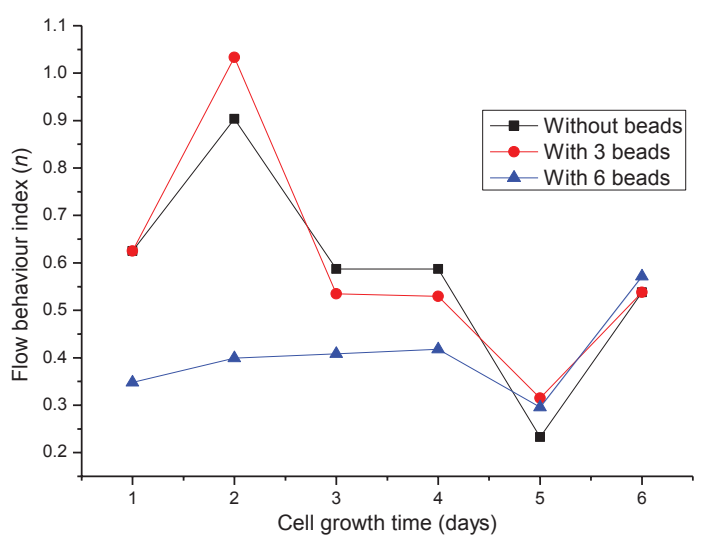

Fig. 7 - Variations in flow behavior index (n) of the power-law fluid model as cell growth occurs with or without glass beads was becoming more shear thinning. Such rheological behavior indicated the role of physical and chemical stimulation or enhanced morphological differentiation that demands better mass transfer within the submerged culture ${ }^{13-15}$. The dispersed mycelium network increased the shear stress and shear rate ratio. During the stationary phase, the morphology of $S$. roseosporus was substantially changed in submerged culture with glass beads, because the swollen hyphae in $S$. roseosporus were differentiated into arthrospores due to the rapid secretion of the antibiotic ${ }^{16}$. At low shear stress conditions (i.e. without beads), the consistency index $(K)$ increased up to $0.28 \mathrm{~Pa} \mathrm{~s}^{\mathrm{n}}$ on day 5 , and then decreased to $0.13 \mathrm{~Pa} \mathrm{~s}^{\mathrm{n}}$ on day 6 . This condition was contradicted by the results seen at higher stress conditions, wherein rapid changes in $K$ and $n$ were revealed. The flow behavior index or the power-law index $(n)$ decreased with increasing stress with time, indicating the typical shear thinning of the culture broth due to complex morphological alterations of actinomycetes ${ }^{17}$. The rheological data changed with the growth phases of the microorganism. Morphological alterations lead to viscosity of the broth, increased respiration rate and the need for proper oxygen mass transfer leading to stress conditions. This stress condition eventually has an adverse effect on the bioprocess or fermentative production of the antibiotic ${ }^{18}$. Therefore, a vivid insight into the rheological pattern of the microorganism would help to track the nature of the fermentative broth, and hence take up measures to increase the mass transfer rate through various process strategies.

\section{Conclusion}

The rheological models used in this study were applied to the experimentally recorded shear stressshear rate data characterizing the flow behavior. Standing in better agreement with the experimental data and keeping consistent with theories, the power law model has done equally well. The present study explored the rheological properties of fermentation broth of $S$. roseosporus, which was influenced by the cell growth and cell differentiation. The morphological changes in the biomass were studied by regulating stress conditions. The non-Newtonian characteristics of the broth confirmed that cells were vigorously differentiated and thus rendered the broth ever more viscous. This kind of viscous nature might hinder mass transfer and production phenomenon of the essential secondary metabolite in the end. The applicability of the power law model would also help to further investigate the changes in volumetric oxygen mass transfer rate with the consistency index. Daptomy- 
cin is indeed a life-saving drug, but has a cost-intensive production process. Thus, this preliminary effort assures that strategic regulation of the hydrodynamics of the fermentation broth can ensure enhanced biomass formation and lead to enhanced production.

\section{References}

1. Eisenstein, B. I., Oleson, F. B., Baltz, R. H., Daptomycin: from the mountain to the clinic, with essential help from Francis Tally, MD, Clinical Infectious Diseases 50 (2010) S10. doi: https://doi.org/10.1086/647938

2. Huber, F. M., Pieper, R. L., Mertz, F. P., Sporulation of Streptomyces roseosporus in submerged culture, Journal of Industrial Microbiology 2 (1987) 235. doi: https://doi.org/10.1007/BF01569545

3. Olsvik, E. S., Kristiansen, B., Influence of oxygen tension, biomass concentration, and specific growth rate on the rheological properties of a filamentous fermentation broth, Biotechnology and Bioengineering 40 (1992) 1293. doi: https://doi.org/10.1002/bit.260401102

4. Riley, G. L., Tucker, K. G., Paul, G. C., Thomas, C. R., Effect of biomass concentration and mycelial morphology on fermentation broth rheology, Biotechnology and Bioengineering 68 (2000) 160.

doi: https://doi.org/10.1002/(SICI)1097-0290(20000420)68:2 $<160:$ :AID-BIT5>3.0.CO; 2-P

5. Petersen, N., Stocks, S., Gernaey, K. V., Multivariate models for prediction of rheological characteristics of filamentous fermentation broth from the size distribution, Biotechnology and Bioengineering 100 (2008) 61 . doi: https://doi.org/10.1002/bit.21732

6. Olsvik, E., Kristiansen, B., Rheology of filamentous fermentations, Biotechnology Advances 12 (1994) 1. doi: https://doi.org/10.1016/0734-9750(94)90288-7

7. Rao, M. A., Flow and functional models for rheological properties of fluid foods, Rheology of Fluid, Semisolid, and Solid Foods, Springer US. (2014) pp. 27-61.

8. Yu, G., Jia, X., Wen, J., Wang, G., Chen, Y., Enhancement of daptomycin production in Streptomyces roseosporus LC51 by manipulation of cofactors concentration in the fermentation culture, World Journal of Microbiology and Biotechnology 27 (2011) 1859.

doi: https://doi.org/10.1007/s11274-010-0644-8
9. Ng, I. S., Ye, C., Zhang, Z., Lu, Y., Jing, K., Daptomycin antibiotic production processes in fed-batch fermentation by Streptomyces roseosporus NRRL11379 with precursor effect and medium optimization, Bioprocess and Biosystems engineering 37 (2014) 415. doi: https://doi.org/10.1007/s00449-013-1007-2

10. Huang, D., Jia, X., Wen, J., Wang, G., Yu, G., Caiyin, Q., Chen, $Y$., Metabolic flux analysis and principal nodes identification for daptomycin production improvement by Streptomyces roseosporus, Appl. Biochem. Biotechnol. 165 (2011) 1725 . doi: https://doi.org/10.1007/s12010-011-9390-0

11. Ghildyal, N. P., Rheological studies on Streptomyces fradiae ScF-5 in submerged fermentation, Journal of Chemical Technology and Biotechnology 38 (1987) 221. doi: https://doi.org/10.1002/jctb.280380402

12. Matsumara, M., Imanaka, T., Yoshida, T., Taguchi, H., Morphological differentiation in relation to cephalosporin C synthesis by cephalosporium acremonium, Journal of Fermentation Ttechnology 58 (1980) 197.

13. Ghojavand, H., Bonakdarpour, B., Heydarian, S. M., Hamedi, J., The inter-relationship between inoculum concentration, morphology, rheology and erythromycin productivity in submerged cultivation of Saccharopolyspora erythraea, Brazilian Journal of Chemical Engineering 28 (2011) 565 . doi: https://doi.org/10.1590/S0104-66322011000400002

14. Lee, H. H., Song, Y. S., Lee, J. Y., Jung, H. W., Kim, S. W., Rheological properties of culture broth of Acremonium chrysogenum M35 in baffled flasks with glass beads, Korea Australia Rheology Journal 22 (2010) 51.

15. Warren, S. J., Keshavarz-Moore, E., Shamlou, P. A., Lilly, M. D., Thomas, C. R., Dixon, K., Rheologies and morphologies of three actinomycetes in submerged culture, Biotechnology and Bioengineering 80 (1995) 80. doi: https://doi.org/10.1002/bit.260450111

16. Chakravarty, I., Kundu, S., Improved production of Daptomycin in an airlift bioreactor by morphologically modified and immobilized cells of Streptomyces roseosporus, AMB Express 6 (1) (2016) 101. doi: https://doi.org/10.1186/s13568-016-0274-0

17. Kumar, R., Rheological characteristics of inulin solution at low concentrations: Effect of temperature and solid content, Journal of food science and technology 52 (9) (2015) 5611. doi: https://doi.org/10.1007/s13197-014-1671-5

18. Alghmadi, A. Y., Rheological characteristics of Ganoderma applanatum exoploysaccharides, African Journal of Microbiology Research 10 (6) (2016) 147. doi: https://doi.org/10.5897/AJMR2015.7744 